\title{
Protective action of Jania rubens nanoencapsulated algal extract in controlling vegetable oils' rancidity
}

\author{
Yasmin R. Maghraby*1, Mohamed A. Farag ${ }^{2,1}$, and Adham R. Ramadan ${ }^{1}$ \\ ${ }^{1}$ Chemistry Department, The American University in Cairo, AUC Avenue, New Cairo 11835, \\ Egypt \\ ${ }^{2}$ Pharmacognosy Department, College of Pharmacy, Cairo University, Kasr El-Aini St., Cairo \\ 11562, Egypt \\ *Corresponding author email: ymaghraby@aucegypt.edu \\ mohamed.alifarag@aucegypt.edu \\ aramadan@aucegypt.edu
}

Table S1: PS, ZP, PDI and EE \% for the prepared JCNP.

\begin{tabular}{|c|l|l|l|l|}
\hline $\begin{array}{c}\text { Optimized } \\
\text { Formula }\end{array}$ & PS \pm SD $(\mathbf{n m})$ & ZP \pm SD $(\mathbf{m V})$ & PDI \pm SD & EE \% \pm SD \\
\hline F1 & $413.5 \pm 2.12$ & $22.95 \pm 0.51$ & $0.304 \pm 0.01$ & $98.25 \pm 0.21$ \\
\hline F2 & $223.5 \pm 0.71$ & $43.95 \pm 0.36$ & $0.262 \pm 0.01$ & $78.70 \pm 0.28$ \\
\hline F3 & $2430.5 \pm 0.72$ & $11.30 \pm 1.55$ & $0.652 \pm 0.07$ & $98.11 \pm 0.14$ \\
\hline F4 & $277.5 \pm 0.71$ & $32.10 \pm 0.14$ & $0.259 \pm 0.01$ & $88.85 \pm 0.07$ \\
\hline F5 & $230.5 \pm 0.72$ & $29.70 \pm 0.28$ & $0.225 \pm 0.01$ & $95.65 \pm 0.78$ \\
\hline F6 & $214.5 \pm 0.72$ & $30.25 \pm 0.35$ & $0.212 \pm 0.01$ & $80.43 \pm 0.71$ \\
\hline F7 & $173.5 \pm 0.72$ & $29.25 \pm 0.92$ & $0.196 \pm 0.01$ & $93.34 \pm 1.70$ \\
\hline F8 & $167.5 \pm 2.12$ & $28.50 \pm 0.14$ & $0.112 \pm 0.02$ & $90.45 \pm 2.62$ \\
\hline F9 & $2356.5 \pm 3.54$ & $12.65 \pm 0.35$ & $0.561 \pm 0.02$ & $99.35 \pm 0.64$ \\
\hline F10 & $332.5 \pm 0.72$ & $28.25 \pm 10.11$ & $0.394 \pm 0.01$ & $80.53 \pm 1.91$ \\
\hline
\end{tabular}




\begin{tabular}{|l|l|l|l|l|}
\hline F11 & $2239.5 \pm 3.54$ & $13.85 \pm 0.35$ & $0.694 \pm 0.01$ & $98.85 \pm 0.94$ \\
\hline F12 & $206.5 \pm 13.45$ & $33.20 \pm 0.26$ & $0.239 \pm 0.01$ & $85.33 \pm 1.91$ \\
\hline F13 & $175.0 \pm 7.79$ & $40.55 \pm 0.78$ & $0.113 \pm 0.01$ & $83.53 \pm 1.70$ \\
\hline F14 & $816.5 \pm 14.14$ & $19.10 \pm 0.42$ & $0.477 \pm 0.03$ & $99.37 \pm 0.21$ \\
\hline F15 & $187.5 \pm 9.19$ & $39.90 \pm 1.83$ & $0.134 \pm 0.02$ & $86.35 \pm 3.18$ \\
\hline F16 & $802.0 \pm 0.71$ & $18.65 \pm 0.92$ & $0.462 \pm 0.01$ & $98.32 \pm 0.28$ \\
\hline F17 & $2586.5 \pm 21.20$ & $13.35 \pm 1.01$ & $0.745 \pm 0.06$ & $99.65 \pm 0.21$ \\
\hline F18 & $229.5 \pm 53.10$ & $36.90 \pm 2.26$ & $0.238 \pm 0.05$ & $87.42 \pm 0.02$ \\
\hline F19 & $2090.5 \pm 6.37$ & $14.55 \pm 1.49$ & $0.645 \pm 0.06$ & $99.15 \pm 0.07$ \\
\hline F20 & $180.0 \pm 82.73$ & $34.30 \pm 2.26$ & $0.171 \pm 0.01$ & $77.60 \pm 0.42$ \\
\hline F21 & $189.6 \pm 2.83$ & $43.10 \pm 0.57$ & $0.128 \pm 0.02$ & $86.94 \pm 0.26$ \\
\hline F22 & $1350.5 \pm 2.89$ & $17.10 \pm 0.28$ & $0.472 \pm 0.02$ & $98.18 \pm 0.26$ \\
\hline F23 & $174.5 \pm 0.72$ & $40.2 \pm 2.69$ & $0.166 \pm 0.03$ & $80.95 \pm 1.06$ \\
\hline F24 & $1708.5 \pm 3.54$ & $18.25 \pm 0.78$ & $0.372 \pm 0.18$ & $99.5 \pm 0.28$ \\
\hline F25 & $232 \pm 2.12$ & $27.85 \pm 0.64$ & $0.2165 \pm 0.01$ & $99.3 \pm 0.57$ \\
\hline F26 & $230.5 \pm 1.41$ & $28.55 \pm 0.64$ & $0.205 \pm 0.01$ & $98.35 \pm 0.21$ \\
\hline F27 & $235 \pm 1.45$ & $30.05 \pm 0.35$ & $0.2155 \pm 0.01$ & $97.8 \pm 0.42$ \\
\hline
\end{tabular}

Table S2: Model summary statistics of quadratic models for tested responses, constrains for optimization of JCNP formula and the predicted and observed values of the responses.

\begin{tabular}{|c|c|c|c|c|c|c|c|}
\hline Responses & $\mathbf{r}^{\mathbf{2}}$ & Adjusted $\mathbf{r}^{\mathbf{2}}$ & Prediction $\mathbf{r}^{\mathbf{2}}$ & Constrains & Predicted & Observed & $\begin{array}{c}\mathbf{9 5 \%} \\
\text { prediction } \\
\text { interval }\end{array}$ \\
\hline $\begin{array}{c}\mathrm{Y}_{1}: \mathrm{PS} \\
(\mathrm{nm})\end{array}$ & 0.8810 & 0.8480 & $\begin{array}{c}0.7770 \\
(0.8117)^{\mathrm{a}}\end{array}$ & Minimize & $\mathbf{1 4 5}$ & $\mathbf{1 6 1} \pm \mathbf{2 . 8 7}$ & $35.77-254.23$ \\
\hline $\begin{array}{c}\mathrm{Y}_{2}: \mathrm{ZP} \\
(\mathrm{mV})\end{array}$ & 0.9232 & 0.8956 & $\begin{array}{c}0.8475 \\
(0.8831)^{\mathrm{a}}\end{array}$ & Maximize & $\mathbf{3 0 . 5 7}$ & $\mathbf{3 1 . 2} \pm \mathbf{0 . 8 7}$ & $28.07-33.06$ \\
\hline $\mathrm{Y}_{3}: \mathrm{PDI}$ & 0.8612 & 0.8114 & $\begin{array}{c}0.7235 \\
(0.7617)^{\mathrm{a}}\end{array}$ & Minimize & $\mathbf{0 . 1 9 6}$ & $\mathbf{0 . 2 1 1} \pm \mathbf{0 . 0 7}$ & $0.131-0.263$ \\
\hline
\end{tabular}




\begin{tabular}{|c|c|c|c|c|c|c|c|}
\hline $\begin{array}{c}\mathrm{Y}_{4}: \mathrm{EE} \\
(\%)\end{array}$ & 0.9010 & 0.8654 & 0.8031 & Maximize & $\mathbf{9 9 . 6}$ & $\mathbf{9 9 . 7} \pm \mathbf{4 . 8 7}$ & $98.9-99.9$ \\
\hline
\end{tabular}

a Reduced model prediction $\mathrm{r}^{2}$.

Table S3: Composition of the $3^{3}$ BBD for JCNP.

\begin{tabular}{|c|c|c|c|c|}
\hline \multirow{2}{*}{$\begin{array}{l}\text { Optimized } \\
\text { Formula }\end{array}$} & \multicolumn{4}{|c|}{ Factors levels in actual values } \\
\hline & CS (w/v \%) & TPP (w/v \%) & HT (min) & HS (rpm) [x 1000] \\
\hline$\overline{\mathrm{F} 1}$ & 0.10 & 0.60 & 5 & 12 \\
\hline $\mathrm{F} 2$ & 0.40 & 0.60 & 5 & 12 \\
\hline F3 & 0.10 & 1.40 & 5 & 12 \\
\hline F4 & 0.40 & 1.40 & 5 & 12 \\
\hline F5 & 0.25 & 1.00 & 3 & 6 \\
\hline F6 & 0.25 & 1.00 & 7 & 6 \\
\hline F7 & 0.25 & 1.00 & 3 & 18 \\
\hline F8 & 0.25 & 1.00 & 7 & 18 \\
\hline F9 & 0.10 & 1.00 & 5 & 6 \\
\hline F10 & 0.40 & 1.00 & 5 & 6 \\
\hline F11 & 0.10 & 1.00 & 5 & 18 \\
\hline F12 & 0.40 & 1.00 & 5 & 18 \\
\hline F13 & 0.25 & 0.60 & 3 & 12 \\
\hline F14 & 0.25 & 1.40 & 3 & 12 \\
\hline F15 & 0.25 & 0.60 & 7 & 12 \\
\hline F16 & 0.25 & 1.40 & 7 & 12 \\
\hline F17 & 0.10 & 1.00 & 3 & 12 \\
\hline F18 & 0.40 & 1.00 & 3 & 12 \\
\hline F19 & 0.10 & 1.00 & 7 & 12 \\
\hline F20 & 0.40 & 1.00 & 7 & 12 \\
\hline F21 & 0.25 & 0.60 & 5 & 6 \\
\hline F22 & 0.25 & 1.40 & 5 & 6 \\
\hline
\end{tabular}




\begin{tabular}{|l|l|l|l|l|}
\hline F23 & 0.25 & 0.60 & 5 & 18 \\
\hline F24 & 0.25 & 1.40 & 5 & 18 \\
\hline F25 & 0.25 & 1.00 & 5 & 12 \\
\hline F26 & 0.25 & 1.00 & 5 & 12 \\
\hline F27 & 0.25 & 1.00 & 5 & 12 \\
\hline
\end{tabular}

